\title{
Degenerate equations in a diffusion-precipitation model for clogging porous media
}

\author{
RAPHAEL SCHULZ \\ Friedrich-Alexander Universität Erlangen-Nürnberg, Department of Mathematics, \\ Cauerstraße 11, 91058 Erlangen, Germany \\ email: raphael.schulz@math.fau.de
}

(Received 30 July 2019; revised 25 October 2019; accepted 25 November 2019;

first published online 18 December 2019)

\begin{abstract}
In this article, we consider diffusive transport of a reactive substance in a saturated porous medium including variable porosity. Thereby, the evolution of the microstructure is caused by precipitation of the transported substance. We are particularly interested in analysing the model when the equations degenerate due to clogging. Introducing an appropriate weighted function space, we are able to handle the degeneracy and obtain analytical results for the transport equation. Also the decay behaviour of this solution with respect to the porosity is investigated. There a restriction on the decay order is assumed, that is, besides low initial concentration also dense precipitation leads to possible high decay. We obtain nonnegativity and boundedness for the weak solution to the transport equation. Moreover, we study an ordinary differential equation (ODE) describing the change of porosity. Thereby, the control of an appropriate weighted norm of the gradient of the porosity is crucial for the analysis of the transport equation. In order to obtain global in time solutions to the overall coupled system, we apply a fixed point argument. The problem is solved for substantially degenerating hydrodynamic parameters.
\end{abstract}

Key words: Evolving porous media, degenerate equation, clogging, weighted spaces, decay behaviour

2010 Mathematics Subject Classification: 35D30, 35K65, 76R50

\section{Introcuction}

Traditionally, a porous medium is characterised by a rigid porous matrix. Recently, the integration of an evolving porous matrix caused by diverse heterogeneous reactions, e.g., crystal precipitation or biofilm growth, has attracted increased interest. Such precipitants or biofilms attach on the surface of the solid matrix and thus occupy pore space. These attachments lead to a changeable solid-liquid interface and affect significantly the diffusivity of the porous medium; they also influence the mass transport of dissolved substances within the pores.

A striking feature of the geometrical changes of the solid-liquid interface at the pore scale is that they have a strong influence on the macroscopic quantities [11]. From a practical point of view, e.g., for the sake of computational feasibility, an upscaled (averaged) model at the macroscale is of major importance compared to the pore-scale model. An extension of formal 
two-scale asymptotic expansion to a level set framework to capturing the evolving solid-liquid interface was introduced in [18]. Besides others, this method was recently applied to locally periodic porous media [19], drug delivery systems [10], non-isothermal media [3] and biofilm growth $[13,14]$.

Let $\Omega \subset \mathbb{R}^{n}, n=2,3$, be an open and bounded domain. In this article, we consider diffusive transport of a reactive substance in a saturated porous medium including variable porosity described by a system of coupled partial differential equations (PDEs). In [12], an effective, non-linear diffusion equation with degenerating coefficients coupled to an ODE for the porosity was derived by two-scale asymptotic expansion in a level set framework. This modelled system of PDEs is analysed in the following. We consider the diffusive transport equation [12, (2.3a)-(2.3b)]

$$
\begin{aligned}
\theta \partial_{t} c-\nabla \cdot \mathbb{D}(\theta) \nabla c & =\left(\frac{c}{\rho}-1\right) f(c, \theta) & & \text { in } \Omega \times(0, T) \\
c & =0 & & \text { on } \partial \Omega \times(0, T) \\
c(., 0) & =c_{0} & & \text { in } \Omega,
\end{aligned}
$$

with the substrate concentration $c$, porosity $\theta \in[0,1)$, constant density $\rho>0$ of the precipitation, reaction rate $f$ and the diffusivity $\mathbb{D}$ (depending on $\theta$ ). Here homogeneous boundary condition and the initial data $c_{0}$ are assumed. The precipitation reaction rate is given by $f(c, \theta):=\bar{\sigma}(\theta) c_{+}$ with $c_{+}:=\max \{0, c\}$ and the function $\bar{\sigma}=\max \left\{0, \theta^{\sigma}\right\}, \sigma \geq 1$. Assuming the reaction $f(c, \theta)$ takes place on the fluid-solid interface (at the microscale), $\bar{\sigma}(\theta)$ describes the corresponding specific surface depending on the porosity $\theta$, cf. e.g., [12, 13, 18].

We aim to have a geometry of the microstructure that may be parametrised by a single parameter, which is represented by the porosity. In such a situation, the hyperbolic level set equation then reduces to an ordinary differential equation (ODE) describing the change of this parameter, cf. [12]. Such an assumption is reasonable since the reaction rate $f$ causing the change of the microstructure occurs uniformly along the fluid-solid interface, i.e., it does not depend on microscopic values. The evolution of the porosity $\theta$ caused by precipitation is given via the following ODE:

$$
\begin{aligned}
\partial_{t} \theta & =-\frac{1}{\rho} f(c, \theta) & & \text { in } \Omega \times(0, T) \\
\theta(., 0) & =\theta_{0} & & \text { in } \Omega .
\end{aligned}
$$

In terms of homogenisation in non-rigid porous media, the literature offers only a small number of analytical results: In [9, 19], upscaling of an advection-diffusion(-reaction) system in a locally periodic medium, including low and high diffusivities, was considered. An effective model describing biofilm growth in porous media was already derived and analysed (up to possible clogging) in [13], but it neglects chemotactical effects completely, which were recently involved in $[14,15]$. The upscaled diffusion-precipitation model (1.1) was considered in [12]. In case of non-degenerating coefficients, global existence or existence up to possible closure of some pores of unique strong solutions was shown. Following up this research, in the current article, we are particularly interested in analysing the model (1.1) when the equations degenerate due to clogging.

Thus far, however, little attention has been paid to the investigation of degenerate transport equations in evolving porous media due to clogging effects, whose analytical investigation is hardly ever available. In applications, e.g., biofouling, clogging phenomena actually appear and 
thus are of particular interest. In [1], degeneracies arising in linear elliptic equations describing two-phase mixtures, e.g. partially melted materials, were managed by an appropriate scaling of the unknowns. There the existence and uniqueness of a solution over the entire domain were shown using a stabilised variational formulation. First, investigations of the behaviour of upscaled equations describing saturated evolving porous media close to clogging can be found in [4]. In [16], the porosity $\theta: \Omega \times(0, T) \rightarrow[0,1)$ was assumed to be a given function, where the degenerate case $\theta(x, t)=0$ was of particular interest and thus admissible. The degeneracy was handled and analytical results were obtained by introducing appropriate weighted function spaces and including the degenerate parameters as weights. In more detail, the non-vanishing parts of the hydrodynamic parameters were proposed to belong to the Muckenhoupt class $A_{2}$.

The current article contributes to this point of degenerate PDEs caused by clogging phenomena in saturated evolving porous media. We will analyse the system (1.1) possible degenerating for $\theta=0$. In this respect, the obtained results extend the knowledge on diffusion-precipitation models presented in or based on $[12,18]$ and actually allow the investigation of clogging processes.

Modelling substrate transporting the effective diffusivity $\mathbb{D}$ is the essential input since it contains all the information that is specific for the considered porous medium. However, this effective tensor is very difficult to characterise in (natural) porous media - even if they are assumed to be represented by scalars. Consequently, formulae in terms of simple features of the porous medium, e.g., the porosity, are frequently used [11]. Along this line, porositydiffusivity models such as linear relations or power laws are often used, even for more general situations. In this work, it is essential to focus on this hydrodynamic parameter when the underlying microstructure clogs and hence the porosity $\theta$ degenerates. Thus, the diffusivity $\mathbb{D}:[0,1) \rightarrow[0, \infty)$ is assumed to be scalar-valued, monotonously increasing map depending on the porosity $\theta$. For the sake of simplicity, it is reasonable to assume that the diffusivity satisfies $\mathbb{D}(\theta)=\theta^{d}$ for some $d \geq 1$. The case $d<1$ is usually not of interest for applications since there exist analytically derived bounds (Voigt-Reiss bound $\mathbb{D}(\theta) \lesssim \theta$, Hashin-Shtrikman bound $\left.\mathbb{D}(\theta) \lesssim \frac{n-1}{n-\theta} \theta\right)$ for the effective diffusion, cf. $[6,11]$.

In fact, the restriction that the diffusivity $\mathbb{D}(\theta)$ degenerates only for $\theta=0$ is generally not reasonable for an arbitrary geometric setting. Depending on the underlying geometry of the medium's microstructure, the diffusivity may already vanish for a positive critical porosity $\theta_{c}>0$, cf. [11]. However, assuming $\theta_{c}=0$ avoids technical excesses due to possible postclogging precipitation processes. Moreover, this assumption will be crucial for Theorem 2.3 focusing on the decay behaviour of the substrates concentration $c$.

The objective of this article is the analytical investigation of the coupled PDE-ODE model (1.1). In this work, it is essential to focus on the effective diffusivity $\mathbb{D}$ when the underlying microstructure clogs and hence the porosity $\theta$ vanishes. Finally, in Section 2, we consider a weak formulation of (1.1a) also in the context of weighted function spaces and obtain by an adjusted Rothe method a unique, nonnegative and bounded solution. We are particularly interested in the decay behaviour of this solution with respect to the given porosity, cf. Theorem 2.3. In Section 3, we investigate the ODE (1.1b) describing the change of porosity due to precipitation. Moreover, the gradient of $\theta$ needs to be studied in more detail, cf. Lemma 3.1. Finally, in Section 4, we apply a fixed point method to solve the overall coupled model (1.1). Therefore, in contrast to [12], the model (1.1) is solved even for substantially degenerating hydrodynamic parameters. 


\section{Analysis for the transport equation (1.1a)}

\subsection{Existence of weak solutions to the transport equation}

Let $\Omega$ be a bounded Lipschitz domain and $\Omega_{T}:=\Omega \times(0, T)$. In the following, the norm of the Banach space $L^{q}(\Omega), q \in[1, \infty]$ is denoted by $\|.\|_{q}$. The rest of the norms occurring below are denoted intuitively. Throughout this paper, $C$ describes positive constants, where the value may differ across occasions. The indices in the constant $C(\cdot, \ldots, \cdot)$ indicate the dependence of parameters.

Since the main objective of this article is solving the coupled system (1.1), we will apply a fixed point procedure in Section 4 such that in each iteration step approximative equations are solved. Therefore, it is useful at this point to modify the equation (1.1a) slightly, i.e., we replace the right-hand side by $\left(\frac{\tilde{c}_{+}}{\rho}-1\right) f(c, \theta)$ with $\tilde{c}_{+}:=\max \{0, \tilde{c}\}$ for a given function $\tilde{c} \in L^{2}\left(0, T ; H_{0}^{1}(\Omega)\right)$ satisfying $\sup _{t \in(0, T)}\|\tilde{c}(t)\|_{\infty} \leq\left\|c_{0}\right\|_{\infty}$. In this section, we analyse this slightly modified diffusive transport equation for a given porosity function $\theta$. The porosity $\theta: \Omega_{T} \rightarrow[0,1)$ is assumed to be decreasing in time, and $\theta \in W^{1, \infty}\left(0, T ; L^{\infty}(\Omega)\right)$ with the decay property of the gradient

$$
\theta^{\frac{d}{2}-1} \nabla \theta \in L^{\infty}\left(0, T ; L^{2}(\Omega)\right)
$$

In order to investigate (1.1a), it is reasonable to define the linear space

$$
V_{\mathbb{D}}(\Omega):=\left\{u \in L^{2}(\Omega): \mathbb{D}(\theta)^{\frac{1}{2}} \nabla u \in\left(L^{2}(\Omega)\right)^{n} \text { and } u=0 \text { on } \partial \Omega\right\}
$$

with the corresponding inner product

$$
(u, v)_{V_{\mathbb{D}}}:=(u, v)_{2}+\left(\mathbb{D}(\theta)^{\frac{1}{2}} \nabla u, \mathbb{D}(\theta)^{\frac{1}{2}} \nabla v\right)_{2},
$$

cf. [1]. Here, $(., .)_{2}$ denotes the inner product of $L^{2}(\Omega)$.

Lemma 2.1 Let the condition (2.1) be satisfied. Then, the space $V_{\mathbb{D}}(\Omega)$ equipped with the above inner product is a Hilbert space.

Proof We proceed similarly to the proof of [1, Lemma 3.1]. It suffices to verify completeness of $V_{\mathbb{D}}(\Omega)$. Thus, let $\left(u_{k}\right)_{k \in \mathbb{N}} \subset V_{\mathbb{D}}(\Omega)$ be a Cauchy sequence, i.e.,

$$
\left\|u_{k}-u_{n}\right\|_{V_{\mathbb{D}}}^{2}=\left\|u_{k}-u_{n}\right\|_{2}^{2}+\left\|\mathbb{D}(\theta)^{\frac{1}{2}} \nabla\left(u_{k}-u_{n}\right)\right\|_{2}^{2} \stackrel{k, n \rightarrow \infty}{\longrightarrow} 0 .
$$

The completeness of $L^{2}(\Omega)$ implies the convergence of the sequences $\left(u_{k}\right)_{k \in \mathbb{N}}$ and $\left(\mathbb{D}(\theta)^{\frac{1}{2}} \nabla u_{k}\right)_{k \in \mathbb{N}}$ in $L^{2}(\Omega)$, i.e., $u_{k} \rightarrow u \in L^{2}(\Omega)$ and $\mathbb{D}(\theta)^{\frac{1}{2}} \nabla u_{k} \rightarrow \psi \in L^{2}(\Omega)$ as $k \rightarrow \infty$. Testing the sequence $\left(\mathbb{D}(\theta)^{\frac{1}{2}} \nabla u_{k}\right)_{k \in \mathbb{N}}$ with a smooth function $\phi \in C_{0}^{\infty}(\Omega)$ yields

$$
\begin{aligned}
\left(\mathbb{D}(\theta)^{\frac{1}{2}} \nabla u_{k}, \phi\right)_{2} & =-\left(u_{k}, \nabla \cdot\left(\mathbb{D}(\theta)^{\frac{1}{2}} \phi\right)\right)_{2} \\
& =-\left(u_{k}, \frac{1}{2} \mathbb{D}(\theta)^{-\frac{1}{2}} \mathbb{D}^{\prime}(\theta) \nabla \theta \phi\right)_{2}+\left(u_{k}, \mathbb{D}(\theta)^{\frac{1}{2}} \nabla \cdot \phi\right)_{2}
\end{aligned}
$$


where the condition (2.1) ensures that $\mathbb{D}(\theta)^{-\frac{1}{2}} \mathbb{D}^{\prime}(\theta) \nabla \theta$ belongs to $L^{2}(\Omega)$. Due to $L^{2}$-convergence of $\left(u_{k}\right)_{k \in \mathbb{N}}$, the right-hand side converges to

$$
\begin{gathered}
-\left(u, \frac{1}{2} \mathbb{D}(\theta)^{-\frac{1}{2}} \mathbb{D}^{\prime}(\theta) \nabla \theta \phi\right)_{2}+\left(u, \mathbb{D}(\theta)^{\frac{1}{2}} \nabla \cdot \phi\right)_{2} \\
=-\left(u, \nabla \cdot\left(\mathbb{D}(\theta)^{\frac{1}{2}} \phi\right)\right)_{2}=\left(\mathbb{D}(\theta)^{\frac{1}{2}} \nabla u, \phi\right)_{2} .
\end{gathered}
$$

Finally, we have $\psi=\mathbb{D}(\theta)^{\frac{1}{2}} \nabla u$ which completes the proof.

In the following, we search for weak solutions

$$
c \in \mathcal{X}_{\theta}:=\left\{L^{2}\left(0, T ; V_{\mathbb{D}}(\Omega)\right) \mid \theta \partial_{t} c \in L^{2}\left(0, T ;\left(V_{\mathbb{D}}(\Omega)\right)^{*}\right)\right\},
$$

where $\left(V_{\mathbb{D}}(\Omega)\right)^{*}$ denotes the dual space of $V_{\mathbb{D}}(\Omega)$. That means a function $c \in \mathcal{X}_{\theta}$ solves the slightly modified transport equation for given $\tilde{c}$ and $\theta$ in a weak sense if

$$
\left\langle\theta \partial_{t} c, \varphi\right\rangle_{V_{\mathbb{D}}^{*}, V_{\mathbb{D}}}+\int_{\Omega} \mathbb{D}(\theta) \nabla c \nabla \varphi=\int_{\Omega}\left(\frac{\tilde{c}_{+}}{\rho}-1\right) f(c, \theta) \varphi,
$$

for all test functions $\varphi \in V_{\mathbb{D}}(\Omega)$. Here $\langle., .\rangle_{V_{\mathbb{D}}^{*}, V_{\mathbb{D}}}$ describes the dual product of $\left(V_{\mathbb{D}}(\Omega)\right)^{*}$ and $V_{\mathbb{D}}(\Omega)$.

To obtain a weak solution of (2.3) with respect to the underlying Hilbert space $V_{\mathbb{D}}(\Omega)$, we apply an adjusted Rothe method similar to [16] and approximate the time derivative $\partial_{t} c$ in (1.1a) by the difference quotient. For this, it is helpful to assume besides conditions on $\theta$, as there are (2.1) and $\partial_{t} \theta \leq 0$ due to precipitation, also a smallness assumption on the initial data $c_{0}$.

Theorem 2.2 Let $\theta \in W^{1, \infty}\left(0, T ; L^{\infty}(\Omega)\right)$ be given and satisfy besides (2.1) also $\theta(x, t) \in[0,1)$, $\|\theta(t)\|_{\infty}>0$, and $\partial_{t} \theta(x, t) \leq 0$ for a.e. $(x, t) \in \Omega_{T}$. Morever, we assume $0 \leq c_{0} \in L^{\infty}(\Omega)$ with $\left\|c_{0}\right\|_{\infty} \leq \rho$. Then there exists a nonnegative unique weak solution $c \in \mathcal{X}_{\theta}$ to (2.3).

Proof In the following, the proof is divided into four main steps:

Semidiscretisation by Rothe's method: Let the interval $[0, T]$ be decomposed in $N \in \mathbb{N}$ equidistant subintervals $\left[t_{k}, t_{k+1}\right]$ with $t_{k}=k \cdot \Delta t, \Delta t=\frac{T}{N}$. Then, $c_{k+1}$ is assumed to be a solution to the discretised (and slightly modified) equation

$$
\theta_{k+1} \frac{c_{k+1}-c_{k}}{\Delta t}-\nabla \cdot\left(\mathbb{D}\left(\theta_{k+1}\right) \nabla c_{k+1}\right)=\left(\frac{1}{\rho} \tilde{c}_{k+1,+}-1\right) f\left(c_{k+1}, \theta_{k+1}\right)-\frac{1}{N} c_{k+1}
$$

for given $c_{k} \in V_{\mathbb{D}}(\Omega), \tilde{c}_{k+1,+}:=\frac{1}{\Delta t} \int_{t_{k}}^{t_{k}+1} \tilde{c}_{+}$and $\theta_{k+1}:=\frac{1}{\Delta t} \int_{t_{k}}^{t_{k}+1} \theta$ as the average of the porosity within the interval $\left[t_{k}, t_{k+1}\right]$. This turns with $c_{k,+}:=\max \left\{0, c_{k}\right\}$ to the elliptic equation

$$
\begin{aligned}
-\nabla \cdot\left(\mathbb{D}\left(\theta_{k+1}\right) \nabla c_{k+1}\right) & +\left[\frac{\theta_{k+1}}{\Delta t}+\left(1-\frac{1}{\rho} \tilde{c}_{k+1,+}\right) \theta_{k+1}^{\sigma}+\frac{1}{N}\right] c_{k+1} \\
& =\frac{1}{\Delta t} \theta_{k+1} c_{k} .
\end{aligned}
$$

Besides the discretisation in time compared to (1.1a), we have also incorporated the additional term $\frac{1}{N} c_{k+1}$ to ensure ellipticity of the problem with respect to $V_{\mathbb{D}}(\Omega)$. Integration by parts yields the weak formulation 


$$
\begin{aligned}
a_{k}\left(c_{k+1}, \varphi\right):= & \left.\int_{\Omega}\left(\mathbb{D}\left(\theta_{k+1}\right)^{\frac{1}{2}} \nabla c_{k+1}\right)\right)\left(\mathbb{D}\left(\theta_{k+1}\right)^{\frac{1}{2}} \nabla \varphi\right) \\
& \quad+\int_{\Omega}\left[\frac{\theta_{k+1}}{\Delta t}+\left(1-\frac{1}{\rho} \tilde{c}_{k+1,+}\right) \theta_{k+1}^{\sigma} c_{k,+}+\frac{1}{N}\right] c_{k+1} \varphi \\
= & \int_{\Omega} \frac{1}{\Delta t} \theta_{k+1} c_{k} \varphi=: b_{k}(\varphi)
\end{aligned}
$$

for all test functions $\varphi \in V_{\mathbb{D}}(\Omega)$. In order to obtain ellipticity of the bilinearform $a_{k}$ with respect to $V_{\mathbb{D}}(\Omega)$, it is helpful to assume the following smallness assumption on $\tilde{c}_{k+1,+}$ :

$$
0 \leq 1-\frac{1}{\rho}\left\|\tilde{c}_{k+1,+}\right\|_{\infty}, \quad \text { i.e., }\left\|\tilde{c}_{k+1,+}\right\|_{\infty} \leq \rho .
$$

This assumption will also be needed in Section 2.2 to prove boundedness of the solution $c$. Then the bilinearform $a_{k}$ is $V_{\mathbb{D}}$-elliptic and continuous. i.e.,

$$
\begin{gathered}
a_{k}(\varphi, \varphi) \geq\left\|\mathbb{D}\left(\theta_{k+1}\right)^{\frac{1}{2}} \nabla \varphi\right\|_{2}^{2}+\frac{1}{N}\|\varphi\|_{2}^{2} \geq \frac{1}{N}\|\varphi\|_{V_{\mathbb{D}}}^{2}, \\
\left|a_{k}(\varphi, \psi)\right| \leq\left\|\mathbb{D}\left(\theta_{k+1}\right)^{\frac{1}{2}} \nabla \varphi\right\|_{2}\left\|\mathbb{D}\left(\theta_{k+1}\right)^{\frac{1}{2}} \nabla \psi\right\|_{2} \\
+\left[\frac{1}{\Delta t}+2+\frac{1}{N}\right]\|\varphi\|_{2}\|\psi\|_{2},
\end{gathered}
$$

and the linearform $b_{k}$ is continuous, i.e.,

$$
|b(\varphi)| \leq \frac{1}{\Delta t}\left\|c_{k}\right\|_{2}\|\varphi\|_{2}
$$

for all $\varphi, \psi \in V_{\mathbb{D}}(\Omega)$. Therefore, for all $k=0, \ldots, N-1$, the theorem of Lax-Milgram yields a unique solution $c_{k+1} \in V_{\mathbb{D}}(\Omega)$ satisfying (2.5).

Boundedness and uniform $L^{2}$-estimate: Since $\partial_{t} \theta \leq 0$ and hence $\theta_{k+1} \leq \theta_{k}$, we have $\left\|\theta_{k+1}^{\frac{1}{2}} c_{k}\right\|_{2} \leq\left\|\theta_{k}^{\frac{1}{2}} c_{k}\right\|_{2}$. Therefore, testing (2.5) with the solution $c_{k+1}$ leads with Young's inequality to the following energy estimate in the weaker seminorm $\left\|\theta_{k+1}^{\frac{1}{2}} \cdot\right\|_{2}$ :

$$
\left.\frac{1}{2 \Delta t}\left(\left\|\theta_{k+1}^{\frac{1}{2}} c_{k+1}\right\|_{2}^{2}-\left\|\theta_{k}^{\frac{1}{2}} c_{k}\right\|_{2}^{2}\right)+\| \mathbb{D}\left(\theta_{k+1}\right)^{\frac{1}{2}} \nabla c_{k+1}\right)\left\|_{2}^{2}+\frac{1}{N}\right\| c_{k+1} \|_{2}^{2} \leq 0 .
$$

Summation over all $k=0, \ldots, N-1$ leads to

$$
\left.\frac{1}{2}\left\|\theta_{N}^{\frac{1}{2}} c_{N}\right\|_{2}^{2}+\Delta t \sum_{k=1}^{N} \| \mathbb{D}\left(\theta_{k}\right)^{\frac{1}{2}} \nabla c_{k}\right)\left\|_{2}^{2}+\frac{\Delta t}{N} \sum_{k=1}^{N}\right\| c_{k}\left\|_{2}^{2} \leq \frac{1}{2}\right\| \theta_{0}^{\frac{1}{2}} c_{0} \|_{2}^{2} .
$$

In order to obtain a solution $c$ belonging to $\mathcal{X}_{\theta}$, an estimate of the $L^{2}$-norm of $c_{k+1}$ is needed uniform with respect to $\Delta t$ and $N$. Therefore, more generally than above, let us now test (2.5) with $\left|c_{k+1}\right|^{2 \alpha} c_{k+1}, \alpha \in \mathbb{N}_{0}$

$$
\begin{gathered}
\int_{\Omega} \mathbb{D}\left(\theta_{k+1}\right) \nabla c_{k+1} \nabla\left(\left|c_{k+1}\right|^{2 \alpha} c_{k+1}\right)+\int_{\Omega}\left[\frac{\theta_{k+1}}{\Delta t}+\left(1-\frac{1}{\rho} \tilde{c}_{k+1,+}\right) \theta_{k+1}^{\sigma}+\frac{1}{N}\right] c_{k+1}^{2 \alpha+2} \\
=\int_{\Omega} \frac{1}{\Delta t} \theta_{k+1} c_{k}\left|c_{k+1}\right|^{2 \alpha} c_{k+1} .
\end{gathered}
$$


Here, the first summand on the left-hand side satisfies with $a:=2 \alpha+2$ the equation

$$
\int_{\Omega} \mathbb{D}\left(\theta_{k+1}\right) \nabla c_{k+1} \nabla\left(\left|c_{k+1}\right|^{2 \alpha} c_{k+1}\right)=(a-1)\left\|\mathbb{D}\left(\theta_{k+1}\right)^{\frac{1}{2}} c_{k+1}^{\alpha} \nabla c_{k+1}\right\|_{2}^{2} .
$$

Since $a$ is an even natural number, the integrand $\left(1-\frac{1}{\rho} \tilde{c}_{k+1,+}\right) \theta_{k+1}^{\sigma} c_{k+1}^{a}$ is nonnegative because of (2.6) and thus can be neglected for the further estimates. This leads to the inequality

$$
\begin{aligned}
\frac{1}{\Delta t}\left\|\theta_{k+1}^{\frac{1}{a}} c_{k+1}\right\|_{a}^{a}+(a-1)\left\|\mathbb{D}\left(\theta_{k+1}\right)^{\frac{1}{2}} c_{k+1}^{\alpha} \nabla c_{k+1}\right\|_{2}^{2} & \leq \frac{1}{\Delta t}\left\|\theta_{k+1}^{\frac{1}{a}} c_{k}\right\|_{a}\left\|\theta_{k+1}^{\frac{1}{a}} c_{k+1}\right\|_{a}^{a-1} \\
& \leq \frac{1}{\Delta t}\left\|\theta_{k}^{\frac{1}{a}} c_{k}\right\|_{a}\left\|\theta_{k+1}^{\frac{1}{a}} c_{k+1}\right\|_{a}^{a-1} .
\end{aligned}
$$

Finally, Young's inequality

$$
\left\|\theta_{k}^{\frac{1}{a}} c_{k}\right\|_{a}\left\|\theta_{k+1}^{\frac{1}{a}} c_{k+1}\right\|_{a}^{a-1} \leq \frac{1}{a}\left\|\theta_{k}^{\frac{1}{a}} c_{k}\right\|_{a}^{a}+\frac{a-1}{a}\left\|\theta_{k+1}^{\frac{1}{a}} c_{k+1}\right\|_{a}^{a}
$$

implies

$$
\frac{1}{a}\left\|\theta_{k+1}^{\frac{1}{a}} c_{k+1}\right\|_{a}^{a}+(a-1) \Delta t\left\|\mathbb{D}\left(\theta_{k+1}\right)^{\frac{1}{2}} c_{k+1}^{\alpha} \nabla c_{k+1}\right\|_{2}^{2} \leq \frac{1}{a}\left\|\theta_{k}^{\frac{1}{a}} c_{k}\right\|_{a}^{a}
$$

In particular, this yields the boundedness of $c_{k+1}$ with

$$
\left\|\theta_{k+1}^{\frac{1}{a}} c_{k+1}\right\|_{a} \leq\left\|\theta_{k}^{\frac{1}{a}} c_{k}\right\|_{a} \leq\left\|\theta_{k}\right\|_{1}^{\frac{1}{a}}\left\|c_{k}\right\|_{\infty}
$$

if the boundedness of $c_{k}$ is supposed. Let $\Omega_{\theta}:=\operatorname{supp} \theta_{k+1}$ denote the support of $\theta_{k+1}$. Then there obviously holds

$$
\left\|\theta_{k+1}^{\frac{1}{a}} c_{k+1}\right\|_{a, \Omega_{\theta}} \leq\left\|\theta_{k+1}^{\frac{1}{a}} c_{k+1}\right\|_{a}
$$

which yields $c_{k+1} \in L^{\infty}\left(\Omega_{\theta}\right)$ in the limit $a \rightarrow \infty$. We obtain by testing (2.5) with a function $\varphi \in V_{\mathbb{D}}(\Omega)$ such that $\operatorname{supp} \varphi \subset \Omega \backslash \Omega_{\theta}$

$$
\int_{\Omega \backslash \Omega_{\theta}}\left[\left(1-\frac{1}{\rho} \tilde{c}_{k+1,+}\right) \theta_{k+1}^{\sigma}+\frac{1}{N}\right] c_{k+1} \varphi=a_{k}\left(c_{k+1}, \varphi\right)=b_{k}(\varphi)=0,
$$

and thus $c_{k+1}=0$ in $\Omega \backslash \Omega_{\theta}$. However, this means $c_{k+1} \in L^{\infty}(\Omega)$ with

$$
\left\|c_{k+1}\right\|_{\infty} \leq\left\|c_{k}\right\|_{\infty} \leq\left\|c_{0}\right\|_{\infty} \leq \rho
$$

and justifies the assumed boundedness of $c_{k}$ by induction. Moreover, this evidently implies $\left\|c_{k+1}\right\|_{2} \leq|\Omega|^{\frac{1}{2}} \rho$ for all $k=0, \ldots, N-1$ and thus together with (2.7) a $N$-uniform energy estimate with respect to the Hilbert space $V_{\mathbb{D}}(\Omega)$

$$
\left\|c_{N}\right\|_{2}^{2}+\Delta t \sum_{k=1}^{N}\left\|\mathbb{D}\left(\theta_{k}\right)^{\frac{1}{2}} \nabla c_{k}\right\|_{2}^{2} \leq|\Omega| \rho^{2}+\frac{1}{2}\left\|\theta_{0}^{\frac{1}{2}} c_{0}\right\|_{2}^{2} .
$$

Convergence for $N \rightarrow \infty$ : Let us now define the functions $c^{N}$ and $\bar{c}^{N}$ on the time interval $[0, T]$ by constant and linear interpolation of $c_{0}, c_{1}, \ldots, c_{N}$, respectively, i.e.,

$$
\begin{array}{ll}
c^{N}(t):=c_{k+1} & \text { for } t \in\left(t_{k}, t_{k+1}\right], \\
\bar{c}^{N}(t):=(1-\mu) c_{k}+\mu c_{k+1} & \text { for } t=(1-\mu) t_{k}+\mu t_{k+1}, \mu \in[0,1] .
\end{array}
$$


In the following, we show that subsequences of these functions converge to the same limit satisfying (2.5). In particular, for $t \in\left(t_{k}, t_{k+1}\right)$, there holds $\partial_{t} \bar{c}^{N}(t)=\frac{c_{k+1}-c_{k}}{\Delta t}$. Besides these functions, we define further $\tilde{c}_{+}^{N}(t):=\tilde{c}_{k+1,+}$ and $\theta^{N}(t):=\theta_{k+1}$ for $t \in\left(t_{k}, t_{k+1}\right]$. Owing to (2.10), the functions $c^{N}, \mathbb{D}\left(\theta^{N}\right) \nabla c^{N}$ and $\bar{c}^{N}$ are uniformly bounded in the function space $L^{2}\left(0, T ; L^{2}(\Omega)\right)$ :

$$
\begin{gathered}
\left.\int_{0}^{T}\left(\left\|c^{N}\right\|_{2}^{2}+\left\|\mathbb{D}\left(\theta^{N}\right)^{\frac{1}{2}} \nabla c^{N}\right\|_{2}^{2}\right) d t=\sum_{k=1}^{N} \int_{t_{k-1}}^{t_{k}}\left(\left\|c_{k}\right\|_{2}^{2}+\| \mathbb{D}\left(\theta_{k}\right)^{\frac{1}{2}} \nabla c_{k}\right) \|_{2}^{2}\right) d t \\
\leq T|\Omega| \rho^{2}+\frac{1}{2}\left\|\theta_{0}^{\frac{1}{2}} c_{0}\right\|_{2}^{2}, \\
\int_{0}^{T}\left\|\bar{c}^{N}\right\|_{2}^{2} d t \leq 2 \Delta t \sum_{k=1}^{N} \int_{0}^{1}\left((1-\mu)\left\|c_{k-1}\right\|_{2}^{2}+\mu\left\|c_{k}\right\|_{2}^{2}\right) d \mu \leq 2 T|\Omega| \rho^{2},
\end{gathered}
$$

i.e., there are subsequences denoted again by $\left(c^{N}\right)_{N \in \mathbb{N}}$ and $\left(\bar{c}^{N}\right)_{N \in \mathbb{N}}$ which converge weakly to some limits $c, \bar{c} \in L^{2}\left(0, T ; L^{2}(\Omega)\right)$, respectively. Also the weak time derivative of $\bar{c}^{N}$ belongs to $L^{2}\left(0, T ; L^{2}(\Omega)\right)$ since

$$
\int_{0}^{T}\left\|\partial_{t} \bar{c}^{N}\right\|_{2}^{2} d t \leq 2 \frac{1}{\Delta t} \sum_{k=1}^{N} \int_{t_{k-1}}^{t_{k}}\left(\left\|c_{k}\right\|_{2}^{2}+\left\|c_{k-1}\right\|_{2}^{2}\right) d t \leq 4 N|\Omega| \rho^{2} .
$$

In what follows, we verify the identity of the limits $c=\bar{c}$ : Testing $\bar{c}^{N}$ with a smooth function $\zeta \in C_{0}^{\infty}\left(\Omega_{T}\right)$ leads to

$$
\begin{aligned}
& \int_{0}^{T}\left(\bar{c}^{N}(t), \zeta(t)\right)_{2} d t \\
& \quad=\Delta t \sum_{k=1}^{N} \int_{0}^{1}\left((1-\mu) c_{k-1}+\mu c_{k}, \zeta\left((1-\mu) t_{k-1}+\mu t_{k}\right)\right)_{2} d \mu \\
& \quad=\Delta t \sum_{k=1}^{N}\left(c_{k}, \zeta_{k}\right)_{2}+\frac{\Delta t}{2}\left(\left(c_{0}, \zeta_{0}\right)_{2}-\left(c_{N}, \zeta_{N}\right)_{2}\right)
\end{aligned}
$$

where $\zeta_{k}:=\zeta\left(t_{k}\right)$ and the term $\frac{\Delta t}{2}\left(\left(c_{0}, \zeta_{0}\right)_{2}-\left(c_{N}, \zeta_{N}\right)_{2}\right)$ is of order $\mathcal{O}\left(\frac{1}{N}\right)$. On the other hand, we have

$$
\begin{aligned}
& \left|\int_{0}^{T}\left(c^{N}(t), \zeta(t)\right)_{2} d t-\Delta t \sum_{k=1}^{N}\left(c_{k}, \zeta_{k}\right)_{2}\right| \leq \sum_{k=1}^{N} \int_{t_{k-1}}^{t_{k}}\left|\left(c_{k}, \zeta(t)-\zeta_{k}\right)_{2}\right| d t \\
& \quad \leq \Delta t \sum_{k=1}^{N} \int_{t_{k-1}}^{t_{k}}\left|\left(c_{k}, \frac{\zeta(t)-\zeta_{k}}{t-t_{k}}\right)_{2}\right| d t \leq \Delta t^{2} C\left(\left\|\partial_{t} \zeta\right\|_{L^{\infty}\left(\Omega_{T}\right)}\right) \sum_{k=1}^{N}\left\|c_{k}\right\|_{2} \\
& \quad \leq \frac{1}{N} T^{2} C\left(\left\|\partial_{t} \zeta\right\|_{L^{\infty}\left(\Omega_{T}\right)}\right)|\Omega|^{\frac{1}{2}} \rho \stackrel{N \rightarrow \infty}{\longrightarrow} 0,
\end{aligned}
$$

which implies the identity of the limits $c$ and $\bar{c}$. 
Limit function solves (2.3): Applying a smooth function $\zeta \in C_{0}^{\infty}\left(\Omega_{T}\right)$ to the diffusive term $\mathbb{D}\left(\theta^{N}\right)^{\frac{1}{2}} \nabla c^{N} \in L^{2}\left(0, T ; L^{2}(\Omega)\right)$, cf. (2.11), leads to the following convergence in the sense of distributions, cf. proof of Lemma 2.1:

$$
\begin{aligned}
-\left\langle\mathbb{D}\left(\theta^{N}\right)^{\frac{1}{2}} \nabla c^{N}, \zeta\right\rangle & =\left(c^{N}, \nabla \cdot\left(\mathbb{D}\left(\theta^{N}\right)^{\frac{1}{2}} \zeta\right)\right)_{L^{2}\left(L^{2}\right)} \\
& \stackrel{N \rightarrow \infty}{\longrightarrow}\left(c, \nabla \cdot\left(\mathbb{D}(\theta)^{\frac{1}{2}} \zeta\right)\right)_{L^{2}\left(L^{2}\right)}=-\left\langle\mathbb{D}(\theta)^{\frac{1}{2}} \nabla c, \zeta\right\rangle,
\end{aligned}
$$

where $(., .)_{L^{2}\left(L^{2}\right)}$ denotes the inner product of $L^{2}\left(0, T ; L^{2}(\Omega)\right)$. The additional assumption (2.1) on the gradient of $\theta$, i.e., $\nabla\left(\mathbb{D}(\theta)^{\frac{1}{2}}\right) \in L^{\infty}\left(0, T ; L^{2}(\Omega)\right)$, ensures convergence in $L^{2}\left(L^{2}\right)$-sense. Moreover, Lebesgue's differentiation theorem [5, Corollary 2.1.16] yields the convergence of $\theta^{N}(x, t)$ to $\theta(x, t)$ for a.e. $(x, t) \in \Omega_{T}$. Since the sequence $\left(\mathbb{D}\left(\theta^{N}\right)^{\frac{1}{2}} \nabla c^{N}\right)_{N \in \mathbb{N}}$ is also uniformly bounded in $L^{2}\left(0, T ; L^{2}(\Omega)\right)$, cf. (2.11), again a subsequence converges weakly and thus $\mathbb{D}\left(\theta^{N}\right)^{\frac{1}{2}} \nabla c^{N} \rightarrow \mathbb{D}(\theta)^{\frac{1}{2}} \nabla c$. Therefore, the limit $c$ satisfies $\mathbb{D}(\theta)^{\frac{1}{2}} \nabla c \in L^{2}\left(0, T ; L^{2}(\Omega)\right)$.

Applying the test function $\zeta \in C_{0}^{\infty}\left(\Omega_{T}\right)$ above to the term associated with the time derivative leads to

$$
\begin{aligned}
\left\langle\theta^{N} \partial_{t} \bar{c}^{N}, \zeta\right\rangle & =-\left(\bar{c}^{N}, \partial_{t}\left(\theta^{N} \zeta\right)\right)_{L^{2}\left(L^{2}\right)} \\
& \stackrel{N \rightarrow \infty}{\longrightarrow}-\left(c, \partial_{t}(\theta \zeta)\right)_{L^{2}\left(L^{2}\right)}=\left\langle\theta \partial_{t} c, \zeta\right\rangle
\end{aligned}
$$

Analogously, we conclude due to the assumptions on $f$ that the remaining term corresponding to the precipitation rate converges also to its temporal continuation. The auxiliary term $-\frac{1}{N} c^{N}$ actually vanishes in the limit as $N \rightarrow \infty$.

However, due to the time discretisation (2.4), there holds for a.e. $t \in(\Delta t, T)$

$$
\theta^{N} \partial_{t} \bar{c}^{N}-\nabla \cdot\left(\mathbb{D}\left(\theta^{N}\right) \nabla c^{N}\right)=\left(\frac{1}{\rho} \tilde{c}_{+}^{N}-1\right) f\left(c^{N}, \theta^{N}\right)-\frac{1}{N} c^{N}
$$

Finally, since each single summand converges, the limit function $c$ in fact satisfies the weak formulation (2.3).

It remains to deduce an appropriate inequality corresponding to the time derivative:

$$
\begin{gathered}
\left\|\theta \partial_{t} c\right\|_{L^{2}\left(V_{\mathbb{D}}^{*}\right)}^{2}=\int_{0}^{T}\left(\sup _{\|\varphi\|_{V_{\mathbb{D}}}=1}\left|\left\langle\theta \partial_{t} c, \varphi\right\rangle_{V_{\mathbb{D}}^{*}, V_{\mathbb{D}}}\right|\right)^{2} \\
\leq \int_{0}^{T}\left(\sup _{\|\varphi\|_{V_{\mathbb{D}}}=1}\left[\|c\|_{V_{\mathbb{D}}}+2\|c\|_{2}\right]\|\varphi\|_{V_{\mathbb{D}}}\right)^{2},
\end{gathered}
$$

i.e., $\theta \partial_{t} c \in L^{2}\left(0, T ;\left(V_{\mathbb{D}}(\Omega)\right)^{*}\right)$. Moreover, the spatio-temporal distributional equation

$$
\begin{aligned}
\left\langle\partial_{t}(\theta c), \zeta\right\rangle & =-\left(c, \theta \partial_{t} \zeta\right)_{L^{2}\left(L^{2}\right)}=-\left(c, \partial_{t}(\theta \zeta)\right)_{L^{2}\left(L^{2}\right)}+\left(c,\left(\partial_{t} \theta\right) \zeta\right)_{L^{2}\left(L^{2}\right)} \\
& =\left\langle\theta \partial_{t} c, \zeta\right\rangle+\left\langle\left(\partial_{t} \theta\right) c, \zeta\right\rangle
\end{aligned}
$$

holds for all $\zeta \in C_{0}^{\infty}\left(\Omega_{T}\right)$ and thus $\partial_{t}(\theta c)$ also belongs to $L^{2}\left(0, T ;\left(V_{\mathbb{D}}(\Omega)\right)^{*}\right)$. 
Finally, the solution $c$ takes the initial value $c_{0} \in L^{\infty}(\Omega)$ in the following weak sense: applying a test function $\zeta \in C_{0}^{\infty}(\Omega \times[0, T))$ to $\theta \partial_{t} c \in L^{2}\left(0, T ;\left(V_{\mathbb{D}}(\Omega)\right)^{*}\right)$ yields

$$
\begin{aligned}
& \int_{0}^{T}\left(\left\langle\left(\theta \partial_{t} c\right)(t), \zeta(t)\right\rangle_{V_{\mathbb{D}}^{*}, V_{\mathbb{D}}}+\left(c(t), \partial_{t}(\theta \zeta)(t)\right)_{2}\right) d t \\
& \quad=\lim _{N \rightarrow \infty} \int_{0}^{T}\left(\left\langle\left(\theta^{N} \partial_{t} \bar{c}^{N}\right)(t), \zeta(t)\right\rangle_{V_{\mathbb{D}}^{*}, V_{\mathbb{D}}}+\left(\bar{c}^{N}(t), \partial_{t}\left(\theta^{N} \zeta\right)(t)\right)_{2}\right) d t \\
& \quad=-\left(c_{0}, \theta_{0} \zeta(0)\right)_{2},
\end{aligned}
$$

where the last equality holds owing to the piecewise affine linearity of $\bar{c}^{N}$ (i.e. $\bar{c}^{N}(0)=c_{0}$ ).

We have already seen above, $c=0$ in $\Omega \backslash \Omega_{\theta}$, i.e., if $\theta=0$. Testing (2.3) with $c_{-}:=\min \{0, c\}$ leads to

$$
\frac{1}{2}\left\|\theta^{\frac{1}{2}} c_{-}(t)\right\|_{2}^{2} \leq \int_{0}^{t} \int_{\Omega}\left(\frac{\tilde{c}_{+}}{\rho}-1\right) f(c, \theta) c_{-}
$$

since $f(c, \theta) c_{-}=0$ and hence $\sup _{t \in(0, T)}\left\|\theta^{\frac{1}{2}} c_{-}(t)\right\|_{2}=0$, i.e., $c(x, t) \geq 0$ for a.e. $(x, t) \in \Omega_{T}$.

The linear structure of (2.3) implies directly the uniqueness of the weak solution $c \in \mathcal{X}_{\theta}$.

\subsection{Decay behaviour of the substrate concentration}

In this section, let the conditions of Theorem 2.2 be satisfied. In what follows, we are interested in boundedness and decay behaviour with respect to $\theta$ of the weak solution $c \in \mathcal{X}_{\theta}$ obtained by the previous theorem.

Theorem 2.3 Let the conditions of Theorem 2.2 be satisfied, where $\theta$ is assumed to be a solution to (3.1) below with $\theta^{-\mu} \nabla \theta \in L^{2}\left(0, T ; L^{2}(\Omega)\right), \mu \in \mathbb{R}$. Additionally, let $\left\|c_{0}\right\|_{\infty} \leq \frac{\rho}{1+p}$ for $p \geq 0$ and $\left\|\theta_{0}^{-p} c_{0}\right\|_{a}<\infty$ for $a=2 \alpha+2, \alpha \in \mathbb{N}_{0}$. If the parameters fulfill the condition $d \geq a p+3-2 \mu$, then the solution $c$ of Theorem 2.2 is bounded and decays of order $p$ with respect to $\theta$ in the $L^{\infty}\left(L^{a}\right)$-norm, i.e.,

$$
\sup _{t \in(0, T)}\|c(t)\|_{\infty} \leq\left\|c_{0}\right\|_{\infty} \quad \text { and } \sup _{t \in(0, T)}\left\|\theta(t)^{-p} c(t)\right\|_{a}<\infty .
$$

Proof Starting point is the application of the test function $\theta^{-v}|c|^{2 \alpha} c, \alpha \in \mathbb{N}_{0}, v \geq 0$, to the weak formulation (2.3), which leads to

$$
\left\langle\theta \partial_{t} c, \theta^{-v}|c|^{2 \alpha} c\right\rangle_{V_{\mathbb{D}}^{*}, V_{\mathbb{D}}}+\int_{\Omega} \mathbb{D}(\theta) \nabla c \nabla\left(\theta^{-v}|c|^{2 \alpha} c\right)=\int_{\Omega}\left(\frac{\tilde{c}_{+}}{\rho}-1\right) f(c, \theta) \theta^{-v}|c|^{2 \alpha} c,
$$

where the both summands on the left-hand side satisfy with $a:=2 \alpha+2$ the identities

$$
\begin{aligned}
\left\langle\theta \partial_{t} c, \theta^{-v}|c|^{2 \alpha} c\right\rangle_{V_{\mathbb{D}}^{*}, V_{\mathbb{D}}} & =\frac{1}{a} \partial_{t}\left\|\theta^{(1-v) / a} c\right\|_{a}^{a}+\frac{1-v}{a} \int_{\Omega} \frac{c}{\rho} f(\tilde{c}, \theta) \theta^{-v}|c|^{2 \alpha} c \\
\int_{\Omega} \mathbb{D}(\theta) \nabla c \nabla\left(\theta^{-v}|c|^{2 \alpha} c\right) & =\int_{\Omega} \mathbb{D}(\theta) \nabla c \nabla\left(\theta^{-v}\right)|c|^{2 \alpha} c+\int_{\Omega} \mathbb{D}(\theta) \nabla c \theta^{-v}(a-1)|c|^{2 \alpha} \nabla c \\
& =(a-1)\left\|\mathbb{D}(\theta)^{\frac{1}{2}} \theta^{-\frac{v}{2}} c^{\alpha} \nabla c\right\|_{2}^{2}-v \int_{\Omega} \mathbb{D}(\theta) \nabla c \theta^{-v-1}|c|^{2 \alpha} c \nabla \theta
\end{aligned}
$$


In the first equation associating with the time derivative of $c$, we have applied (3.1) below, i.e., $\partial_{t} \theta$ is replaced by $-\frac{1}{\rho} f(\tilde{c}, \theta)$ with the function $\tilde{c}$. The term $c f(\tilde{c}, \theta)$ actually coincides by definition of the function $f$ and the nonnegativity of $c$ with $\tilde{c}_{+} f(c, \theta)$. Therefore, we obtain with Young's inequality and $C(a)>0$ (such that the diffusive term on the left-hand side absorbs the corresponding term on the right-hand side)

$$
\begin{aligned}
\frac{1}{a}\left\|\theta(t)^{(1-v) / a} c(t)\right\|_{a}^{a} & +\frac{1}{2}(a-1) \int_{0}^{t}\left\|\mathbb{D}(\theta)^{\frac{1}{2}} \theta^{-\frac{v}{2}} c^{\alpha} \nabla c\right\|_{2}^{2} \leq \frac{1}{a}\left\|\theta_{0}^{(1-v) / a} c_{0}\right\|_{a}^{a} \\
& +v^{2} C(a) \int_{0}^{t}\left\|\mathbb{D}(\theta)^{\frac{1}{2}} \theta^{-\frac{v}{2}-1} c^{\alpha+1} \nabla \theta\right\|_{2}^{2} \\
& +\int_{0}^{t} \int_{\Omega}\left[\frac{\tilde{c}_{+}}{\rho}-\frac{1-v}{a} \frac{\tilde{c}_{+}}{\rho}-1\right] f(c, \theta) \theta^{-v}|c|^{2 \alpha} c
\end{aligned}
$$

Since $\tilde{c}$ is assumed to satisfy $\sup _{t \in(0, T)}\|\tilde{c}(t)\|_{\infty} \leq\left\|c_{0}\right\|_{\infty} \leq \rho$, the negativity of the term within the square bracket is ensured as long as

$$
\tilde{c}_{+}\left(1-\frac{1-v}{a}\right) \leq \rho,
$$

which is fulfilled for $v \leq 1$.

In order to obtain boundedness of $c$, let us at first choose $v:=0$ leading to the following simplification of (2.13):

$$
\frac{1}{a}\left\|\theta(t)^{\frac{1}{a}} c(t)\right\|_{a}^{a}+(a-1) \int_{0}^{t}\left\|\mathbb{D}(\theta)^{\frac{1}{2}} c^{\alpha} \nabla c\right\|_{2}^{2} \leq \frac{1}{a}\left\|\theta_{0}^{\frac{1}{a}} c_{0}\right\|_{a}^{a} .
$$

Thus,

$$
\sup _{t \in(0, T)}\left\|\theta(t)^{\frac{1}{a}} c(t)\right\|_{a} \leq\left\|\theta_{0}^{\frac{1}{a}} c_{0}\right\|_{a}
$$

i.e., $c \in L^{\infty}\left(0, T ; \theta^{-\frac{1}{a}} L^{a}(\Omega)\right)$ for all $a=2 \alpha+2$ and hence the limit for $a \rightarrow \infty$ yields boundedness of $c$ with $\sup _{t}\|c(t)\|_{\infty} \leq\left\|c_{0}\right\|_{\infty}$.

Note that $\left(1-\frac{1-v}{a}\right)$ is larger than 1 for $v>1$, this leads in the following to a stronger restriction on the initial data $c_{0}$. Now let $v:=a p+1$ with $p>0$. This choice of $v$ is helpful to work out the decay of $c$ with respect to $\theta$, i.e., $p$ is the order of decay of $c$. Inequality (2.13) implies

$$
\begin{aligned}
\frac{1}{a}\left\|\theta(t)^{-p} c(t)\right\|_{a}^{a} & +\frac{1}{2}(a-1) \int_{0}^{t}\left\|\mathbb{D}(\theta)^{\frac{1}{2}} \theta^{-\frac{a p+1}{2}} c^{\alpha} \nabla c\right\|_{2}^{2} \leq \frac{1}{a}\left\|\theta_{0}^{-p} c_{0}\right\|_{a}^{a} \\
& +(a p+1)^{2} C(a) \int_{0}^{t}\left\|\mathbb{D}(\theta)^{\frac{1}{2}} \theta^{-\frac{a p+3}{2}} c^{\alpha+1} \nabla \theta\right\|_{2}^{2} \\
& +\int_{0}^{t} \int_{\Omega}\left[(1+p) \frac{\tilde{c}_{+}}{\rho}-1\right] f(c, \theta) \theta^{-v}|c|^{2 \alpha} c
\end{aligned}
$$

where the last summand on the right-hand side is negative (and hence negligible) if the smallness assumption $\left\|c_{0}\right\|_{\infty} \leq \frac{\rho}{1+p}$ is fulfilled. Finally, this yields with $d \geq a p+3-2 \mu$

$$
\begin{aligned}
\frac{1}{a}\left\|\theta(t)^{-p} c(t)\right\|_{a}^{a} & +\frac{1}{2}(a-1) \int_{0}^{t}\left\|\mathbb{D}(\theta)^{\frac{1}{2}} \theta^{-\frac{a p+1}{2}} c^{\alpha} \nabla c\right\|_{2}^{2} \leq \frac{1}{a}\left\|\theta_{0}^{-p} c_{0}\right\|_{a}^{a} \\
& +(a p+1)^{2} C(a) \int_{0}^{t}\left\|\mathbb{D}(\theta) \theta^{-a p-3+2 \mu}\right\|_{\infty}\|c\|_{\infty}^{a}\left\|\theta^{-\mu} \nabla \theta\right\|_{2}^{2},
\end{aligned}
$$


which actually leads to

$$
\sup _{t \in(0, T)}\left\|\theta(t)^{-p} c(t)\right\|_{a}^{a} \leq\left\|\theta_{0}^{-p} c_{0}\right\|_{a}^{a}+a(a p+1)^{2} C(a) \rho^{a}\left\|\theta^{-\mu} \nabla \theta\right\|_{L^{2}\left(L^{2}\right)}^{2} .
$$

This inequality is valid for all $a \geq 2$ satisfying the parameter condition.

Remark We obtained an interesting condition for the upper bound of the order of decay of $c$ since the above smallness assumption changes to $p \leq \frac{\rho}{\left\|c_{0}\right\|_{\infty}}-1$. That means besides low initial concentration also dense precipitation leads to possible high decay. Since in such cases, precipitation causes only small changes in the microstructure and hence in the porosity, the concentration decreases with respect to the porosity rather fast. In contrast, small $\rho$ leads to large changes in the porosity but small changes in the concentration.

\section{Analysis for the porosity}

\subsection{Solvability of the ODE (1.1b)}

Let again $\tilde{c} \in L^{2}\left(0, T ; H_{0}^{1}(\Omega)\right)$ be a given function satisfying $\sup _{t \in(0, T)}\|\tilde{c}\| \leq\left\|c_{0}\right\|_{\infty} \leq \rho$. We slightly modify the original ODE $(1.1 \mathrm{~b})$ in this section by replacing the unknown $c$ of (1.1a) with $\tilde{c}$, i.e., we consider

$$
\int_{\Omega}\left(\partial_{t} \theta\right) \varphi=-\frac{1}{\rho} \int_{\Omega} f(\tilde{c}, \theta) \varphi
$$

for all $\varphi \in L^{2}(\Omega)$. According to the theory of ODEs, there exists a unique absolutely continuous solution $\theta \in \mathcal{Y}:=H^{1}\left(0, T ; L^{2}(\Omega)\right)$ represented by

$$
\theta(t)=\theta_{0}-\frac{1}{\rho} \int_{0}^{t} f(\tilde{c}, \theta)
$$

The assumed positivity property of $f$ implies directly

$$
\theta(x, t) \in\left[0, \theta_{0}(x)\right] \quad \text { for a.e. }(x, t) \in \Omega_{T},
$$

i.e., in particular $\partial_{t} \theta \leq 0$. Furthermore, we have $\theta \in \mathcal{Y} \cap W^{1, \infty}\left(0, T ; L^{\infty}(\Omega)\right)$ with

$$
\begin{gathered}
\sup _{t \in(0, T)}\|\theta(t)\|_{\infty}+\sup _{t \in(0, T)}\left\|\partial_{t} \theta(t)\right\|_{\infty} \leq\left\|\theta_{0}\right\|_{\infty}+\frac{1}{\rho} \sup _{t \in(0, T)}\|f(\tilde{c}, \theta)(t)\|_{\infty} \\
\leq\left\|\theta_{0}\right\|_{\infty}+\frac{1}{\rho}\left\|c_{0}\right\|_{\infty}\left\|\theta_{0}\right\|_{\infty}^{\sigma} \leq 2 .
\end{gathered}
$$

\subsection{Decay behaviour of the gradient of $\theta$}

The regularity of $\tilde{c}$ with respect to $x$ basically carries over to $\theta$ since the spatial variable $x \in \Omega$ is only a parameter in the $\operatorname{ODE}(1.1 \mathrm{~b})$. Therefore, since $\tilde{c}$ is assumed to belong to $L^{2}\left(0, T ; H_{0}^{1}(\Omega)\right)$, it is reasonable that also $\nabla \theta$ can be estimated in $L^{2}\left(0, T ; L^{2}(\Omega)\right)$. For this, we test (3.1) with $\nabla \theta$ which leads to

$$
\frac{1}{2}\|\nabla \theta(t)\|_{2}^{2} \leq \frac{1}{2}\left\|\nabla \theta_{0}\right\|_{2}^{2}+\sigma \int_{0}^{T}\|\theta\|_{\infty}^{\sigma-1}\|\tilde{c}\|_{\infty}\|\nabla \theta\|_{2}^{2}+\int_{0}^{T}\left\|\theta^{\sigma} \nabla \tilde{c}\right\|_{2}\|\nabla \theta\|_{2}
$$


and hence with Gronwall's Lemma

$$
\sup _{t \in(0, T)}\|\nabla \theta(t)\|_{2} \leq\left(\left\|\nabla \theta_{0}\right\|_{2}+\int_{0}^{T}\|\nabla \tilde{c}\|_{2}\right) \cdot \exp \left[\sigma \int_{0}^{T}\|\tilde{c}\|_{\infty}\right]<\infty .
$$

However, since the property $\theta^{\frac{d}{2}-1} \nabla \theta \in L^{\infty}\left(0, T ; L^{2}(\Omega)\right)$ (cf. (2.1)) was crucial for the previous theorems, it is necessary to obtain $L^{\infty}\left(L^{2}\right)$-estimates of the gradient of $\theta$ including an appropriate weight $\theta^{-\mu}, \mu \geq 1-\frac{d}{2}$.

Lemma 3.1 Let the function $\tilde{c} \in L^{2}\left(0, T ; H_{0}^{1}(\Omega)\right) \cap L^{\infty}\left(\Omega_{T}\right)$ and the parameter $\mu \leq \sigma$ be given. The function $\theta \in W^{1, \infty}\left(0, T ; L^{\infty}(\Omega)\right)$ denotes the solution to (3.1), where the initial porosity satisfies $\theta_{0}^{-\mu} \nabla \theta_{0} \in L^{2}(\Omega)$. Then the weighted gradient $\theta^{-\mu} \nabla \theta$ belongs to $L^{\infty}\left(0, T ; L^{2}(\Omega)\right)$.

Proof Let us test (3.1) with $-\nabla \cdot\left(\theta^{-2 \mu} \nabla \theta\right)$

$$
\begin{aligned}
\frac{1}{2} \partial_{t}\left\|\theta^{-\mu} \nabla \theta\right\|_{2}^{2} & =-\mu \int_{\Omega} \theta^{-2 \mu-1} \partial_{t} \theta|\nabla \theta|^{2}+\int_{\Omega} \partial_{t}(\nabla \theta) \theta^{-2 \mu} \nabla \theta \\
& =\frac{\mu}{\rho} \int_{\Omega} \theta^{-2 \mu-1} f(\tilde{c}, \theta)|\nabla \theta|^{2}-\frac{1}{\rho} \int_{\Omega} \nabla f(\tilde{c}, \theta) \theta^{-2 \mu} \nabla \theta .
\end{aligned}
$$

Therefore, integration over time leads to

$$
\begin{aligned}
\frac{1}{2}\left\|\theta^{-\mu}(t) \nabla \theta(t)\right\|_{2}^{2} \leq \frac{1}{2}\left\|\theta_{0}^{-\mu} \nabla \theta_{0}\right\|_{2}^{2} & +\frac{\mu}{\rho} \int_{0}^{t}\left\|\theta^{-1} f(\tilde{c}, \theta)\right\|_{\infty}\left\|\theta^{-\mu} \nabla \theta\right\|_{2}^{2} \\
& +\frac{1}{\rho} \int_{0}^{t}\left\|\theta^{-\mu} \nabla f(\tilde{c}, \theta)\right\|_{2}\left\|\theta^{-\mu} \nabla \theta\right\|_{2} .
\end{aligned}
$$

With $\nabla f(\tilde{c}, \theta)=\nabla\left(\bar{\sigma}(\theta) r \tilde{c}_{+}\right)=\sigma \theta^{\sigma-1}(\nabla \theta) \tilde{c}_{+}+\bar{\sigma}(\theta) \nabla \tilde{c}_{+}$, we obtain

$$
\begin{aligned}
\frac{1}{2} \| \theta^{-\mu}(t) & \nabla \theta(t)\left\|_{2}^{2} \leq \frac{1}{2}\right\| \theta_{0}^{-\mu} \nabla \theta_{0}\left\|_{2}^{2}+\frac{\mu}{\rho} \int_{0}^{t}\right\| \theta^{-1} \bar{\sigma}(\theta) \tilde{c}_{+}\left\|_{\infty}\right\| \theta^{-\mu} \nabla \theta \|_{2}^{2} \\
& +\frac{\sigma}{\rho} \int_{0}^{t}\left\|\theta^{\sigma-1} \tilde{c}_{+}\right\|_{\infty}\left\|\theta^{-\mu} \nabla \theta\right\|_{2}^{2}+\frac{1}{\rho} \int_{0}^{t}\left\|\bar{\sigma}(\theta)\left(\nabla \tilde{c}_{+}\right) \theta^{-\mu}\right\|_{2}\left\|\theta^{-\mu} \nabla \theta\right\|_{2},
\end{aligned}
$$

and finally a finite norm in $L^{\infty}\left(0, T ; L^{2}(\Omega)\right)$ by Gronwall's Lemma

$$
\begin{gathered}
\sup _{t \in(0, T)}\left\|\theta^{-\mu}(t) \nabla \theta(t)\right\|_{2}^{2} \leq\left(\left\|\theta_{0}^{-\mu} \nabla \theta_{0}\right\|_{2}+\frac{1}{\rho} T^{\frac{1}{2}}\left\|\theta^{\sigma-\mu}(t) \nabla \tilde{c}_{+}(t)\right\|_{L^{2}\left(L^{2}\right)}\right)^{2} \\
\times \exp \left[2 \frac{1}{\rho} T(\mu+\sigma) \sup _{t \in(0, T)}\left\|\theta^{\sigma-1}(t) \tilde{c}_{+}(t)\right\|_{\infty}\right]
\end{gathered}
$$

where the assumptions $\sigma \geq 1$ and $\mu \leq \sigma$ on the parameters ensure together with the properties of $\tilde{c}$ the boundedness of the right-hand side.

This result is useful in the context of (2.1), if we choose $\mu \geq 1-\frac{d}{2}$. 


\section{Fixed point argument for the coupled problem (1.1)}

This final section is concerned with the weak solvability of the overall coupled system (1.1). In Section 2, the problem (2.3) was solved with respect to the $\theta$-weighted linear space $\mathcal{X}_{\theta}$. In the following, the underlying function space is assumed to be a complete Bochner space. Thus, we denote the essential solution spaces by

$$
\begin{aligned}
& \mathcal{X}:=L^{2}\left(0, T ; H_{0}^{1}(\Omega)\right) \cap H^{1}\left(0, T ; H^{-1}(\Omega)\right), \\
& \mathcal{Y}:=H^{1}\left(0, T ; L^{2}(\Omega)\right), \quad \text { and } \mathbb{X}:=\mathcal{X} \times \mathcal{Y} .
\end{aligned}
$$

In general, there hold the following inclusions for a bounded porosity weight $\theta$ :

$$
H_{0}^{1}(\Omega) \subset V_{\mathbb{D}}(\Omega) \subset L^{2}(\Omega) \subset\left(V_{\mathbb{D}}\right)^{*}(\Omega) \subset H^{-1}(\Omega),
$$

i.e., $L^{2}\left(0, T ; V_{\mathbb{D}}(\Omega)\right)$ is interpreted as a subspace of the rigid Bochner space $L^{2}\left(0, T ; L^{2}(\Omega)\right)$. The solution $c \in \mathcal{X}_{\theta}$ of Theorem 2.2 belongs also to $\mathcal{X}$ if it lies in $L^{2}\left(0, T ; H_{0}^{1}(\Omega)\right)$. This can be ensured by an additional condition $d \leq 2 p+1$ on the parameters and (2.15). Moreover, we introduce

$$
\tilde{\mathcal{Y}}:=W^{1, \infty}\left(0, T ; L^{\infty}(\Omega)\right) \cap L^{\infty}\left(0, T ; H_{0}^{1}(\Omega)\right) \subset \mathcal{Y},
$$

as well as the closed subspace $\mathcal{N}:=\left\{y \in \tilde{\mathcal{Y}}: \theta^{\frac{d}{2}-1} \nabla \theta \in L^{\infty}\left(0, T ; L^{2}(\Omega)\right)\right\}$. The function space $\tilde{\mathcal{Y}}$ is compactly embedded in $L^{q}\left(0, T ; L^{\infty}(\Omega)\right), q<\infty$, cf. [2].

Definition 4.1 A tuple $(c, \theta) \in \mathbb{X}$ of functions is called a weak solution to the coupled system of equations (1.1), if for all test functions $\left(\varphi_{1}, \varphi_{2}\right) \in H_{0}^{1}(\Omega) \times L^{2}(\Omega)$ and a. e. $t \in(0, T)$ there holds

$$
\begin{aligned}
\left\langle\theta \partial_{t} c, \varphi_{1}\right\rangle_{H^{-1}, H_{0}^{1}} & =-\int_{\Omega} \mathbb{D}(\theta) \nabla c \nabla \varphi_{1}+\int_{\Omega}\left(\frac{c}{\rho}-1\right) f(c, \theta) \varphi_{1}, \\
\int_{\Omega}\left(\partial_{t} \theta\right) \varphi_{2} & =-\frac{1}{\rho} \int_{\Omega} f(c, \theta) \varphi_{2},
\end{aligned}
$$

and if $(c, \theta)$ takes the initial value $\left(c_{0}, \theta_{0}\right) \in L^{2}(\Omega)^{2}$ in the sense

$$
\left|\left(c(t)-c_{0}, \phi\right)_{2}\right|+\left|\left(\theta(t)-\theta_{0}, \phi\right)_{2}\right| \stackrel{t \rightarrow 0}{\longrightarrow} 0,
$$

for all $\phi \in L^{2}(\Omega)$.

Our main result states the existence of a weak solution $(c, \theta) \in \mathbb{X}$ to (1.1) in the sense of Definition 4.1 for any arbitrary interval of time $(0, T), T>0$. Besides the assumptions of Theorem 2.3 and Lemma 3.1 on the involved parameters, additionally $d \leq 2 p+1$ is necessary.

Theorem 4.2 Let the parameters $\sigma, d \geq 1, \mu \geq 1-\frac{d}{2}, p \geq 0$ and $a \in 2 \mathbb{N}$ satisfy the following restrictions:

$$
\mu \leq \sigma \quad \text { and } \quad \text { ap }+3-2 \mu \leq d \leq 2 p+1 .
$$

Moreover, the nonnegative initial data $c_{0}, \theta_{0} \in L^{\infty}(\Omega)$ are assumed to fulfill

$$
\left\|c_{0}\right\|_{\infty} \leq \frac{\rho}{1+p}, \quad\left\|\theta_{0}^{-p} c_{0}\right\|_{a}<\infty, \quad\left\|\theta_{0}\right\|_{\infty}<1 \quad \text { and } \quad\left\|\theta_{0}^{-\mu} \nabla \theta_{0}\right\|_{2}<\infty .
$$


Then for all $T>0$, there exists a nonnegative solution $(c, \theta) \in \mathcal{X} \times \mathcal{N}$ to (4.1) with

$$
\sup _{t \in(0, T)}\|c(t)\|_{\infty} \leq\left\|c_{0}\right\|_{\infty} \quad \text { and } \sup _{t \in(0, T)}\|\theta(t)\|_{\infty} \leq\left\|\theta_{0}\right\|_{\infty} .
$$

In particular, the solution $c$ and the gradient of $\theta$ are decaying of order $p$ in the $L^{\infty}\left(L^{a}\right)$-norm and of order $\mu$ in the $L^{\infty}\left(L^{2}\right)$-norm, respectively.

Proof Let $T>0$ and $M>0$, we define the closed and convex subset

$$
\begin{aligned}
\mathcal{M}:= & \left\{c \in L^{2}\left(0, T ; H_{0}^{1}(\Omega)\right) \cap L^{\infty}\left(\Omega_{T}\right) \mid\|c\|_{L^{2}\left(H^{1}\right)} \leq M, \sup _{t \in(0, T)}\|c(t)\|_{\infty} \leq\left\|c_{0}\right\|_{\infty}\right. \\
& \text { and } \left.c \text { satisfies the initial condition in (1.1a) })_{3}\right\} .
\end{aligned}
$$

of $L^{2}\left(0, T ; H_{0}^{1}(\Omega)\right) \cap L^{\infty}\left(\Omega_{T}\right)$. Since $L^{2}\left(0, T ; H_{0}^{1}(\Omega)\right) \cap L^{\infty}\left(\Omega_{T}\right)$ has a predual space isomporhic to $L^{2}\left(0, T ; H^{-1}(\Omega)\right)+L^{1}\left(\Omega_{T}\right)$, the subset $\mathcal{M}$ is in particular weak*-compact according to the theorem of Banach-Alaoglu-Bourbaki. Now, let $\mathcal{F}: \mathcal{M} \rightarrow \mathcal{M}$ be an operator defined as composition $\mathcal{F}:=\mathcal{F}_{2} \circ \mathcal{F}_{1}$ of two operators

$$
\mathcal{F}_{1}: \mathcal{M} \rightarrow \mathcal{N}, \quad \mathcal{F}_{2}: \mathcal{N} \rightarrow \mathcal{M}
$$

The operator $\mathcal{F}_{1}$ is defined by $\mathcal{F}_{1}(\tilde{c}):=\theta$, where $\theta$ denotes the solution to the equation (3.1) for given $\tilde{c} \in \mathcal{M}$. Moreover, $\mathcal{F}_{2}(\theta):=c$ is the solution to $(2.3)$. Then $(c, \theta)$ takes the initial data $\left(c_{0}, \theta_{0}\right)$ in the sense of $(4.1 \mathrm{c})$.

Well-defined self-map: First, we verify that $\mathcal{F}$ is a well-defined self-map on $\mathcal{M}$. Owing to Section 3.1, the map $\mathcal{F}_{1}$ is well defined on $\mathcal{M}$ and (3.3) yields the estimate

$$
\sup _{t \in(0, T)}\|\theta(t)\|_{\infty}+\sup _{t \in(0, T)}\left\|\partial_{t} \theta(t)\right\|_{\infty} \leq 2 .
$$

Moreover, Lemma 3.2 implies $\theta \in \mathcal{N}$.

Furthermore, Theorem 2.2 guarantees the existence of a solution $c \in \mathcal{X}_{\theta}$ to (2.3) additionally satisfying the $L^{\infty}$-estimate

$$
\sup _{t \in(0, T)}\|c(t)\|_{\infty} \leq\left\|c_{0}\right\|_{\infty} .
$$

Theorem 2.3 leads with the assumed restriction $d \leq 2 p+1$ on the parameters to $c \in$ $L^{2}\left(0, T ; H_{0}^{1}(\Omega)\right.$ since we have in $(2.15)$ for $a=2$

$$
\begin{aligned}
\|\nabla c\|_{L^{2}\left(L^{2}\right)}^{2} & \leq\left\|\mathbb{D}(\theta)^{\frac{1}{2}} \theta^{-p-\frac{1}{2}} \nabla c\right\|_{L^{2}\left(L^{2}\right)}^{2} \\
& \leq\left\|\theta_{0}^{-p} c_{0}\right\|_{2}^{2}+(2 p+1)^{2}\left\|\theta_{0}\right\|_{\infty}^{d-2 p-3+2 \mu}\left\|c_{0}\right\|_{\infty}^{2} \int_{0}^{T}\left\|\theta^{-\mu} \nabla \theta\right\|_{2}^{2} .
\end{aligned}
$$

The norm of the weighted gradient of $\theta$ can be estimated by (3.4) leading actually to

$$
\begin{aligned}
\|\nabla c\|_{L^{2}\left(L^{2}\right)} \leq \| \theta_{0}^{-p} & c_{0}\left\|_{2}+(2 p+1)\right\| \theta_{0}\left\|_{\infty}^{\frac{1}{2}(d-2 p-3+2 \mu)}\right\| c_{0} \|_{\infty} T^{\frac{1}{2}} \\
& \times\left(\left\|\theta_{0}^{-\mu} \nabla \theta_{0}\right\|_{2}+\frac{1}{\rho} T^{\frac{1}{2}}\left\|\theta_{0}\right\|_{\infty}^{\sigma-\mu} M\right) \cdot \exp [T(\mu+\sigma)]
\end{aligned}
$$


such that for sufficiently large $M$ and small $T$, we obtain $\|\nabla c\|_{L^{2}\left(L^{2}\right)} \leq \frac{1}{2} M$. Finally, $c$ belongs to $\mathcal{M}$ and hence the operator $\mathcal{F}$ maps $\mathcal{M}$ into $\mathcal{M}$.

Weak*-continuity: To prove continuity of the operator $\mathcal{F}$ with respect to the weak*-topology, we show first weak*-continuity of $\mathcal{F}_{1}$. Therefore, let $\left(\tilde{c}_{k}\right)_{k \in \mathbb{N}} \subset \mathcal{M}$ be a sequence weak*converging to $\tilde{c} \in \mathcal{M}$. Then, the sequence $\left(\theta_{k}\right)_{k \in \mathbb{N}}=\left(\mathcal{F}_{1}\left(\tilde{c}_{k}\right)\right)_{k \in \mathbb{N}}$ is bounded due to (3.3) and (3.4). Moreover, a subsequence of $\left(\theta_{k}\right)_{k \in \mathbb{N}}$ (denoted again by $\left(\theta_{k}\right)_{k \in \mathbb{N}}$ ) weak*-converges to a limit $\theta \in \mathcal{Y} \cap L^{2}\left(0, T ; H^{1}(\Omega)\right)$. The Lemma of Aubin-Lions implies strong convergence in $L^{2}\left(0, T ; L^{2}(\Omega)\right)$. The limit $\theta$ actually satisfies (3.1) since

$$
\begin{aligned}
& \int_{0}^{T}\left(\partial_{t}\left(\theta_{k}-\theta\right), \varphi\right)_{2} \stackrel{k \rightarrow \infty}{\longrightarrow} 0 \quad \text { and } \\
& \int_{0}^{T}\left(f\left(\tilde{c}_{k}, \theta_{k}\right)-f(\tilde{c}, \theta), \varphi\right)_{2}=\int_{0}^{T}\left(\tilde{c}_{k}\left(\theta_{k}^{\sigma}-\theta^{\sigma}\right), \varphi\right)_{2}+\int_{0}^{T}\left(\left(\tilde{c}_{k}-\tilde{c}\right) \theta^{\sigma}, \varphi\right)_{2} \\
& \quad \leq \sup _{t \in(0, T)}\left\|\tilde{c}_{k}(t)\right\|_{\infty}\left\|\theta_{k}^{\sigma}-\theta^{\sigma}\right\|_{L^{2}\left(L^{2}\right)}\|\varphi\|_{L^{2}\left(L^{2}\right)}+\int_{0}^{T}\left(\left(\tilde{c}_{k}-\tilde{c}\right) \theta^{\sigma}, \varphi\right)_{2} \stackrel{k \rightarrow \infty}{\longrightarrow} 0
\end{aligned}
$$

for all $\varphi \in L^{2}\left(0, T ; L^{2}(\Omega)\right)$. Besides the weak*-convergence of $\left(\tilde{c}_{k}\right)_{k \in \mathbb{N}}$ and the strong $L^{2}\left(L^{2}\right)$ convergence of $\left(\theta_{k}\right)_{k \in \mathbb{N}}$, we have applied in the last step the estimate $\left|\theta_{k}^{\sigma}-\theta^{\sigma}\right| \leq \sigma\left|\theta_{k}-\theta\right|$ since $\theta_{k}, \theta \in(0,1)$.

The sequence $\left(\theta_{k}\right)_{k \in \mathbb{N}}$ defines a further sequence $\left(c_{k}\right)_{k \in \mathbb{N}}=\left(\mathcal{F}_{2}\left(\theta_{k}\right)\right)_{k \in \mathbb{N}}$. The boundedness of this sequence in $L^{2}\left(0, T ; L^{2}(\Omega)\right)$ leads to a weak* convergent subsequence to a limit $c \in$ $L^{2}\left(0, T ; L^{2}(\Omega)\right)$. The weak*-continuity of the operator $\mathcal{F}$ is verified if $c=\mathcal{F}(\tilde{c})$, i.e., if $c$ satisfies (2.3) with respect to $\theta$. Since $\left(c_{k}\right)_{k \in \mathbb{N}} \subset L^{2}\left(0, T ; H_{0}^{1}(\Omega)\right)$, the time derivatives $\theta_{k} \partial_{t} c_{k}$ belong to $L^{2}\left(0, T ; H^{-1}(\Omega)\right)$ and are similarly to $(2.12)$ uniformly bounded. Thus, a subsequence (denoted again by $\left.\left(\theta_{k} \partial_{t} c_{k}\right)_{k \in \mathbb{N}}\right)$ weakly* converges to $\theta \partial_{t} c$. Considering the terms associated with the time derivative, we have for all $\varphi \in L^{2}\left(0, T ; H_{0}^{1}(\Omega)\right)$

$$
\int_{0}^{T}\left(\theta_{k} \partial_{t} c_{k}-\theta \partial_{t} c, \varphi\right)_{2} \stackrel{k \rightarrow \infty}{\longrightarrow} 0 .
$$

The products $\theta_{k} c_{k}, \theta c$ belong also to $L^{2}\left(0, T ; H_{0}^{1}(\Omega)\right)$ and hence the sequence $\left(\theta_{k} c_{k}\right)_{k \in \mathbb{N}}$ is bounded in $\mathcal{X}$, i.e., there is a weak ${ }^{*}$-convergent subsequence (denoted again by $\left(\theta_{k} c_{k}\right)_{k \in \mathbb{N}}$ ) with limit $\theta c$. In particular, $\left(\theta_{k} c_{k}\right)_{k \in \mathbb{N}}$ converges strongly in $L^{2}\left(0, T ; L^{2}(\Omega)\right)$ to $\theta c$. Owing to this strong $L^{2}\left(L^{2}\right)$-convergence, the convergence of the terms in (2.3) associated with the right-hand side also holds true

$$
\begin{aligned}
& \int_{0}^{T}\left(\left(\frac{\tilde{c}_{k,+}}{\rho}-1\right) f\left(c_{k}, \theta_{k}\right)-\left(\frac{\tilde{c}_{+}}{\rho}-1\right) f(c, \theta), \varphi\right)_{2} \\
&=\frac{1}{\rho} \int_{0}^{T}\left(\tilde{c}_{k,+}\left(f\left(c_{k}, \theta_{k}\right)-f(c, \theta)\right), \varphi\right)_{2}+\frac{1}{\rho} \int_{0}^{T}\left(\left(\tilde{c}_{k,+}-\tilde{c}_{+}\right) f(c, \theta), \varphi\right)_{2} \\
&+\int_{0}^{T}\left(f(c, \theta)-f\left(c_{k} \theta_{k}\right), \varphi\right)_{2}
\end{aligned}
$$




$$
\begin{aligned}
\leq\left(\frac{1}{\rho} \sup _{t \in(0, T)}\left\|\tilde{c}_{k,+}(t)\right\|_{\infty}\right. & +1)\left(\left\|\theta_{k} c_{k}-\theta c\right\|_{L^{2}\left(L^{2}\right)}\|\varphi\|_{L^{2}\left(L^{2}\right)}\right. \\
& \left.+\sup _{t \in(0, T)}\|c(t)\|_{\infty}\left\|\theta_{k}^{\sigma-1}-\theta^{\sigma-1}\right\|_{L^{2}\left(L^{2}\right)}\|\varphi\|_{L^{2}\left(L^{2}\right)}\right) \\
& +\frac{1}{\rho}\left|\int_{0}^{T}\left(\left(\tilde{c}_{k,+}-\tilde{c}_{+}\right) f(c, \theta), \varphi\right)_{2}\right| \stackrel{k \rightarrow \infty}{\longrightarrow} 0,
\end{aligned}
$$

for all $\varphi \in L^{2}\left(0, T ; H_{0}^{1}(\Omega)\right)$. Here, it is useful to apply the auxiliary Lemma 4.3 below, ensuring that

$$
\left\|\theta_{k}^{\sigma-1}-\theta^{\sigma-1}\right\|_{L^{2}\left(L^{2}\right)} \stackrel{k \rightarrow \infty}{\longrightarrow} 0
$$

for all $\sigma \geq 1$.

The sequence of gradients $\left(\nabla c_{k}\right)_{k \in \mathbb{N}} \subset L^{2}\left(0, T ; L^{2}(\Omega)\right)$ is uniformly bounded by $\frac{1}{2} M$ and hence entails a weakly* convergent subsequence. Finally, the continuity assumption on $\mathbb{D}$ implies

$$
\begin{aligned}
& \left|\int_{0}^{T}\left(\mathbb{D}\left(\theta_{k}\right) \nabla c_{k}-\mathbb{D}(\theta) \nabla c, \nabla \varphi\right)_{2}\right| \leq\left|\int_{0}^{T}\left(\mathbb{D}(\theta) \nabla\left(c_{k}-c\right), \varphi\right)\right| \\
& \quad+\left\|\mathbb{D}\left(\theta_{k}\right)-\mathbb{D}(\theta)\right\|_{L^{2}\left(L^{2}\right)}\left\|\nabla c_{k}\right\|_{L^{2}\left(L^{2}\right)} \sup _{t \in(0, T)}\|\nabla \varphi(t)\|_{\infty} \stackrel{k \rightarrow \infty}{\longrightarrow} 0
\end{aligned}
$$

for all smooth test functions $\varphi \in C_{0}^{\infty}\left(\Omega_{T}\right)$. The density of $C_{0}^{\infty}\left(\Omega_{T}\right)$ in $L^{2}\left(0, T ; H_{0}^{1}(\Omega)\right)$ leads for an arbitrary test function $\varphi \in L^{2}\left(0, T ; H_{0}^{1}(\Omega)\right)$ and a sequence of smooth functions $\varphi_{\ell} \in C_{0}^{\infty}\left(\Omega_{T}\right)$ converging to $\varphi$ as $\ell \rightarrow \infty$ to the following result. Let $\varepsilon>0$. Since $\left\|\nabla c_{k}\right\|_{L^{2}\left(L^{2}\right)} \leq \frac{1}{2} M$ is uniformly bounded, choosing $\ell$ sufficiently large such that $\left\|\nabla c_{k}\right\|_{L^{2}\left(L^{2}\right)}\left\|\varphi-\varphi_{\ell}\right\|_{L^{2}\left(H^{1}\right)}<\frac{\varepsilon}{2}$ and afterwards choosing $k$ in such a way that

$$
\left\|\mathbb{D}\left(\theta_{k}\right)-\mathbb{D}(\theta)\right\|_{L^{2}\left(L^{2}\right)}\left\|\nabla c_{k}\right\|_{L^{2}\left(L^{2}\right)} \sup _{t \in(0, T)}\left\|\nabla \varphi_{\ell}(t)\right\|_{\infty}<\frac{\varepsilon}{2},
$$

we obtain

$$
\begin{aligned}
\int_{0}^{T}\left(\left(\mathbb{D}\left(\theta_{k}\right)-\mathbb{D}(\theta)\right) \nabla c_{k}, \nabla \varphi\right) \leq\left\|\nabla c_{k}\right\|_{L^{2}\left(L^{2}\right)}\left\|\varphi-\varphi_{\ell}\right\|_{L^{2}\left(H^{1}\right)} \\
+\left\|\mathbb{D}\left(\theta_{k}\right)-\mathbb{D}(\theta)\right\|_{L^{2}\left(L^{2}\right)}\left\|\nabla c_{k}\right\|_{L^{2}\left(L^{2}\right)} \sup _{t \in(0, T)}\left\|\nabla \varphi_{\ell}(t)\right\|_{\infty}<\varepsilon .
\end{aligned}
$$

After all, Schauder's fixed point theorem ensure a solution $c \in \mathcal{X}$ to (4.1a) with the properties derived in Section 2. This implies a solution $\theta \in \mathcal{Y}$ to (4.1b) satisfying the estimates in Section 3. The proof of the nonnegativity of $c$ follows the same line as in Theorem 2.3.

Global in time solution: Let finally $T>0$ be arbitrary. We decompose the time interval $[0, T]$ into $L$ subintervals $0=: T_{0}<T_{1}<\ldots<T_{L}:=T$, where $\left[T_{i-1}, T_{i}\right]$ has to be chosen small enough such that the fixed point procedure above yields the existence of weak solutions $\left(c_{i}, \theta_{i}\right)$ within the $i$-th subinterval corresponding to the initial data $\left(c_{i-1}\left(T_{i}\right), \theta_{i-1}\left(T_{i}\right)\right), i=1, \ldots, L$. Since $c_{i}, \theta_{i} \in \mathbb{X} \hookrightarrow C\left(0, T ; L^{2}(\Omega)\right)$, this successive definition of initial data is well defined. Also the assumptions made on the initial data are satisfied. These solutions are uniformly bounded in $\mathbb{X}$, 
which actually leads to a temporal continuation to a solution $(c, \theta) \in \mathbb{X}$ with respect to the entire interval $[0, T]$.

The additional assumptions $a p+3-2 \mu \leq d \leq 2 p+1$ on the parameters in the previous Theorem imply also the following relation

$$
\mu \geq \frac{1}{2}(a-2) p+1
$$

i.e., in particular $\mu \geq 1$. These several restrictions on the parameters which have to be satisfied do not intersect in an empty set. For instance, the choice $a=2$ and $\sigma=1$ yield $\mu=1$ and hence $d=2 p+1$.

In contrast to Theorem 2.2, standard arguments do not yield uniqueness of the weak solution $(c, \theta) \in \mathbb{X}$. This is besides the strong non-linear structure of $(2.3)$ with respect to $(c, \theta)$ also due to the unsymmetric $\theta$ weights of the time derivative and the diffusion terms, respectively.

The $L^{2}\left(L^{2}\right)$-strong convergence of the sequence $\left(\theta_{k}\right)_{k \in \mathbb{N}}$ to $\theta$ was crucial in the previous proof. It is evident that also powers $\left(\theta_{k}^{a}\right)_{k \in \mathbb{N}}$ of this sequence with $a \geq 1$ converge strongly to $\theta^{a}$. We conclude this section with the following result stating the $L^{2}\left(L^{2}\right)$-convergence of powers with exponents $a \in(0,1)$, which can be verified directly by applying Hölder's inequality.

Lemma 4.3 Let $\sigma \in(1,2)$ and $\left(\theta_{k}\right)_{k \in \mathbb{N}} \subset L^{2}\left(0, T ; L^{2}(\Omega)\right)$ be a sequence strongly converging to $\theta \in L^{2}\left(0, T ; L^{2}(\Omega)\right)$. Moreover, we assume $\theta_{k}, \theta \in[0,1)$ for all $k \in \mathbb{N}$. Then the sequence $\left(\theta_{k}^{\sigma-1}\right)_{k \in \mathbb{N}} \subset L^{2}\left(0, T ; L^{2}(\Omega)\right)$ converges strongly to $\theta^{\sigma-1} \in L^{2}\left(0, T ; L^{2}(\Omega)\right)$.

Proof First, let $\sigma-1 \geq \frac{1}{2}$. In this situation, we have

$$
\begin{aligned}
\| \theta_{k}^{\sigma-1} & -\theta^{\sigma-1} \|_{L^{2}\left(L^{2}\right)}^{2}=\int_{0}^{T} \int_{\Omega}\left|\theta_{k}^{\sigma-1}-\theta^{\sigma-1}\right|^{2} \\
& \leq \int_{0}^{T} \int_{\Omega}\left|\theta_{k}^{\sigma-1}-\theta^{\sigma-1}\right|\left|\theta_{k}^{\sigma-1}+\theta^{\sigma-1}\right|=\left\|\theta_{k}^{2(\sigma-1)}-\theta^{2(\sigma-1)}\right\|_{L^{1}\left(L^{1}\right)} \\
& \leq(T|\Omega|)^{\frac{1}{2}}\left\|\theta_{k}^{2(\sigma-1)}-\theta^{2(\sigma-1)}\right\|_{L^{2}\left(L^{2}\right)} \stackrel{k \rightarrow \infty}{\longrightarrow} 0,
\end{aligned}
$$

since $2(\sigma-1) \geq 1$. If $\sigma-1 \geq \frac{1}{4}$, we obtain

$$
\begin{aligned}
\| \theta_{k}^{\sigma-1} & -\theta^{\sigma-1}\left\|_{L^{2}\left(L^{2}\right)}^{2}=(T|\Omega|)^{\frac{1}{2}}\right\| \theta_{k}^{\sigma-1}-\theta^{\sigma-1} \|_{L^{4}\left(L^{4}\right)}^{2} \\
& \leq(T|\Omega|)^{\frac{1}{2}}\left(\int_{0}^{T} \int_{\Omega}\left|\theta_{k}^{\sigma-1}-\theta^{\sigma-1}\right|^{2}\left|\theta_{k}^{\sigma-1}+\theta^{\sigma-1}\right|^{2}\right)^{\frac{1}{2}} \\
& =(T|\Omega|)^{\frac{1}{2}}\left\|\theta_{k}^{2(\sigma-1)}-\theta^{2(\sigma-1)}\right\|_{L^{2}\left(L^{2}\right)} \stackrel{k \rightarrow \infty}{\longrightarrow} 0
\end{aligned}
$$

since $2(\sigma-1) \geq \frac{1}{2}$. Finally, let $m \in \mathbb{N}$ such that $\sigma-1 \geq \frac{1}{2^{m}}$. Induction over $m$ completes the proof. 


\section{Conclusions}

We analysed the diffusion-precipitation model (1.1) including vanishing porosity. Introducing an appropriate weighted function space, we were able to handle the degeneracy and obtained analytical results for the transport equation, $\mathrm{cf}$. Theorem 2.2. We investigated the decay behaviour of this solution with respect to the porosity in Theorem 2.3. There the restriction on the decay order $p \leq \frac{\rho}{\left\|c_{0}\right\|_{\infty}}-1$ was assumed, i.e., besides low initial concentration also dense precipitation leads to possible high decay. We obtained nonnegativity and boundedness for the weak solution to the transport equation. Moreover, we studied the ODE describing the change of porosity caused by precipitation. Thereby, the control of an appropriate $\theta$-weighted norm of the gradient of the porosity was crucial for the analysis of the transport equation. In order to obtain global in time solutions to the overall coupled system, we applied a fixed point argument. However, in contrast to previous works, e.g., $[12,13,14]$, we were able to solve (1.1) even in case of substantially clogging phenomena.

For sake of simplicity, the diffusivity was proposed to be scalar-valued. In fact, this parameter is a tensor $\mathbb{D}:[0,1) \rightarrow \mathbb{R}^{(n, n)}$ in anisotropic media [11]. Such a generalisation can be done easily since the effective tensor is typically bounded, symmetric and uniformly positive definite [6, 13].

Even though this article is a first step towards rigorous analysis for clogging porous media, a lot of future work needs still to be done in various directions. Since standard arguments do not yield uniqueness of weak solutions to (1.1) due to non-linearity and unsymmetric $\theta$ weights, another approach is necessary. Also fluid flow and advective transport should be incorporated for a more comprehensive model.

The main attention in the current article was being directed to solutions with decaying behaviour. In such a case, i.e., $c \stackrel{\theta \searrow 0}{\longrightarrow} 0$, a constant dissolution rate [7] would dominate the reactions for small $\theta$, i.e., clogging will never appear. Therefore, we neglected the reaction of dissolution and focused on clogging effects. However, for sufficiently small density $\rho$ or large initial concentration $c_{0}$, it may happen that the concentration does not decay with respect to $\theta$ and hence clogging may occur despite a dissolution rate. However, the property $\partial_{t} \theta \leq 0$ (i.e., no dissolution) was crucial in the proof of Theorem 2.2 to derive the discrete energy estimate (2.7) as well as (2.8). Therefore, including dissolution is a demanding task requiring novel analytical approaches.

As already mentioned in the introduction, the analytically justified restriction on the critical porosity $\theta_{c}=0$ is not exhaustive for general geometric settings. In contrast to Theorem 2.3, the concentration $c$ does not necessarily vanish for $\theta \rightarrow \theta_{c}$ if $\theta_{c}>0$ is assumed. Thus, also for $\theta \leq \theta_{c}$ substrate may still precipitate at the solid matrix leading to a post-clogging ODE system. Therefore, also analysis for $\theta_{c}>0$ is of importance and should be done in future.

Finally, besides equation (1.1), also generalised non-linear diffusion problems of the form

$$
\partial_{t} b(u)-\nabla \cdot \mathbb{D}(x, t, u) \nabla u=f(x, t, u) \quad \text { in } \Omega \times(0, T)
$$

are often of relevance for several applications. Thus, it is of interest whether the current results can be extended to such possibly degenerating parabolic equations and in which sense the decay behaviour is reflected. For monotone increasing functions $b$, a $L$-scheme-type linearisation might be useful [8]. 


\section{References}

[1] Arbogast, T. \& TAicher, A. L. (2016) A linear degenerate elliptic equation arising from two-phase mixtures. SIAM J. Numer. Anal. 54(5), 3105-3122.

[2] ARendt, W. \& Kreuter, M. (2018) Mapping theorems for Sobolev-spaces of vector-valued functions. Studia Mathematica 240, 275-299.

[3] Bringedal, C., Berre, I., Pop, I. S. \& RAdU, F. A. (2016) Upscaling of non-isothermal reactive porous media flow with changing porosity. Transp. Porous Med. 114(2), 371-393.

[4] BRINGedAL, C. \& KUMAR, K. (2017) Effective behavior near clogging in upscaled equations for non-isothermal reactive porous media flow. Transp. Porous Med. 120(3), 553-577.

[5] Grafakos, L. (2009) Classical Fourier analysis, Graduate Texts in Mathematics, 2nd edn., vol. 249, Springer, New York.

[6] Jikov, V. V., Kozlov, S. M. \& OleiniK, O. A. (1994) Homogenization of Differential Operators and Integral Functionals, Springer-Verlag, Berlin Heidelberg.

[7] KUMAR, K., POP, I. S. \& RADU, F. A. (2013) Convergence analysis of mixed numerical schemes for reactive flow in a porous medium. SIAM J. Numer. Anal. 51(4), 2283-2308.

[8] Mitra, K. \& Pop, I. S. (2019) A modified $L$-scheme to solve nonlinear diffusion problems. Comput. Math. Appl. 77(6), 1722-1738.

[9] Muntean, A. \& Van Noorden, T. L. (2013) Corrector estimates for the homogenization of a locally periodic medium with areas of low and high diffusivity. European J. Appl. Math. 24, $657-677$.

[10] Ray, N., Van Noorden, T. L., Radu, F. A., Friess, W., Knabner, P. (2013) Drug release from collagen matrices including an evolving microstructure. ZAMM Z. Angew. Math. Mech. 93, $811-822$.

[11] Ray, N., Rupp, A., Schulz, R. \& Knabner, P. (2018) Old and new approaches predicting the diffusion in porous media. Transp. Porous Med. 124(3), 803-824.

[12] Schulz, R., Ray, N., Frank, F., Mahato, H. \& Knabner, P. (2017) Strong solvability up to clogging of an effective diffusion-precipitation model in an evolving porous medium. Eur. J. Appl. Math. 28(2), 179-207.

[13] Schulz, R. \& KNABneR, P. (2017) Derivation and analysis of an effective model for biofilm growth in evolving porous media. Math. Meth. Appl. Sci. 40(8), 2930-2948.

[14] Schulz, R. \& Knabner, P. (2017) An effective model for biofilm growth made by chemotactical bacteria in evolving porous media. SIAM J. Appl. Math. 77(5), 1653-1677.

[15] Schulz, R. (2017) Boundedness in a biofilm-chemotaxis model in evolving porous media. Math. Model. Anal. 22(6), 852-869.

[16] Schulz, R. (2020) Degenerate equations for flow and transport in clogging porous media. J. Math. Anal. Appl. 483(2), 1-24. DOI: 10.1016/j.jmaa.2019.123613.

[17] VAN NOORDEN, T. L. (2007) Crystal precipitation and dissolution in a thin strip, Technical Report, CASA report 30, Eindhoven University of Technology.

[18] VAn Noorden, T. L. (2009) Crystal precipitation and dissolution in a porous medium: Effective equations and numerical experiments. Multiscale Model. Simul. 7, 1220-1236.

[19] VAn Noorden, T. L. \& Muntean, A. (2011) Homogenization of a locally-periodic medium with areas of low and high diffusivity. Eur. J. Appl. Math. 22, 493-516. 